\title{
A qualitative study of the challenges of providing pre-prosthetic rehabilitation in rural South Africa
}

Liezel Ennion and Anton Johannesson

\begin{abstract}
Background: There is a known shortage of rehabilitation staff in rural settings and a sharp increase in the number of lower limb amputations being performed. A lack of adequate pre-prosthetic rehabilitation will result in worse physical and psychological outcomes for a person with a lower limb amputation, and they will not be eligible to be fitted with a prosthesis.
\end{abstract}

Objective: To explore therapists' experiences with providing pre-prosthetic rehabilitation in a rural setting.

Study design: A qualitative descriptive approach was used to collect and analyse data.

Methods: Data were collected from 17 purposively sampled therapists in five district hospitals in a rural community in South Africa. Data were collected in two rounds of focus groups to explore the challenges of providing pre-prosthetic rehabilitation in rural South Africa.

Results: The main themes identified in the study were (1) a lack of government health system support, (2) poor socioeconomic circumstances of patients and (3) cultural factors that influence rehabilitation. These themes all negatively influence the therapists' ability to follow up patients for pre-prosthetic rehabilitation after discharge from hospital. A lack of adequate pre-prosthetic rehabilitation is a substantial barrier to prosthetic fitting in rural South Africa. Patients who do not receive pre-prosthetic rehabilitation have a poorly shaped residuum or other complications such as knee or hip joint contractures which disqualifies them from being referred to prosthetic services.

Conclusion: Therapists involved in this study identified the most important barriers to patients having access to prosthetic services.

\section{Clinical relevance}

Pre-prosthetic rehabilitation provides care of the residuum; maintenance or improvement of physical strength, joint range of motion and referral to a prosthetist. By exploring the challenges known to exist in this service, we can identify potential ways to reduce these barriers and improve the lives of those who use it.

\section{Background}

Prosthetic rehabilitation has proven to be effective in improving a patient's mobility after lower limb amputation (LLA) (referred here as LLA through the ankle and higher) and 
improving quality of life. ${ }^{1-4}$ Physiotherapists lead the pre-prosthetic rehabilitation which provides care of the residuum, issuing of an initial assistive device, maintenance and improvement of physical strength and joint range of motion, balance training and referral to prosthetic services once the individual is ready. 5 However, in a previous study in this setting, a lack of pre-prosthetic rehabilitation was identified as a barrier to prosthetic fitting. ${ }^{6}$ If patients with an LLA have no access to pre-prosthetic rehabilitation services, they were unlikely to be fitted with a prosthesis.

In South Africa, all physiotherapy and occupational therapy students are required to complete a year of compulsory community service before they are recognised as therapists by the Health Professionals Council of South Africa.7 Many of the therapy students are placed in rural settings in order to address the known shortages of health-care staff in underresourced settings. An increasing number of LLAs are being performed in rural settings due to the increase in Type 2 diabetes mellitus. 8 These changes in incidence require increased and sufficient pre- and post-prosthetic rehabilitation services.

To date, no studies have explored the experiences of therapists who provide preprosthetic rehabilitation services in these areas to determine the challenges, in order to inform possible solutions.

The aim of this study was to explore the experiences of rural therapists (Physiotherapists and Occupational therapists) specifically in relation to providing pre-prosthetic rehabilitation in a rural setting in Kwa-Zulu Natal (KZN) province in South Africa (SA).

\section{Challenges relating to prosthetic rehabilitation in developing countries}

Prosthetic rehabilitation and service delivery in developing countries, especially in rural settings, are very challenging.4,9,10 Some of these problems include (1) inaccessibility of healthcare services, (2) a lack of trained rehabilitation personnel to provide rehabilitation services, (3) difficulty attracting and retaining staff and (4) a lack of research and evidence-based practice.4,9-12

\section{Inaccessibility of healthcare services}

A number of barriers limit access to healthcare services in rural areas in South Africa. Financial barriers, distance to healthcare facilities and limited availability of services as well as inadequate public transport were some of the major barriers that have been identified.13 Major rehabilitation centres and therapeutic services are usually located in urban settings and not easily accessible to rural residents. 4

\section{A lack of trained rehabilitation personnel to provide rehabilitation services} According to the World Health Organization (WHO), $0.5 \%$ of the total population in developing countries require orthoses or prostheses. Therefore approximately 30 million people need an estimated 180,000 rehabilitation professionals. 4 In 2005 , only 400 prosthetists were trained annually in all the developing countries in the world, which is 
inadequate to meet the increasing need for prosthetic services in developing countries. ${ }^{11} \mathrm{In}$ 2011, there was only one physiotherapist and less than one occupational therapist per 10,000 members of the total population in SA, most of whom were employed in urban settings. 4 The limited number of therapists in SA, together with their unequal geographical distribution between urban and rural settings, inevitably creates massive unmet rehabilitation needs in rural settings. 12 The understaffing of most rural clinics further affects the magnitude as well as the quality of services that are provided.13,14

\section{Difficulty attracting and retaining staff}

The major shortages of rehabilitation staff in SA, coupled with the difficulty of retaining the few that do work in a rural setting, pose a major challenge to providing rehabilitation services in rural settings. In an attempt to address this rehabilitation staff deficit, mid-level therapists are being trained in a number of developing countries, including SA. 15 Attempts are also being made to recruit students from the rural areas into health programmes at a tertiary level, in the hope that they will return to their own communities to work. 4 Another SA initiative to address staff shortages in rural areas is the community service programme that all health professionals in SA have to complete in order to be able to register with the Health Professionals Council of South Africa. 6

\section{Inappropriate assistive devices}

A significant challenge highlighted in the literature is the lack of appropriate assistive technology or devices for rural settings. ${ }^{10,16}$ Assistive devices include prostheses, wheelchairs and crutches as well as any aid that improves the ability of a person with a disability to function independently.4 A lack of appropriate assistive devices such as elbow crutches will limit mobility of a persons with an LLA while they are on the waiting list to receive a prosthesis. A lack of mobility will negatively impact on their physical strength and predispose them to develop hip and knee joint contractures, which will further reduce their chances of receiving a prosthesis. The WHO calls for appropriate assistive technology that suits the demands of the environment as well as that of the patient, to ensure optimal fit, comfort and function as far as possible. 4

\section{Methods}

\section{Research team and reflexivity}

The first author was qualified with an MSc in Physiotherapy at the time of data collection and conducted the focus group discussions (FGDs) in person. She worked in a similar rural setting in 2005 and was familiar with a couple of the permanent therapists who still worked in the area. The objectives of the study were explained to participants when they signed informed consent to participate in the research. The researcher attempted to remain as unbiased as possible throughout the study but could potentially have introduced a bias by being able to relate to some of the challenges with providing pre-prosthetic rehabilitation in a rural setting. The confirmability of the findings was ensured by asking an independent researcher to perform a data audit and analyse sections of the data to confirm that the themes were appropriate. 


\section{Theoretical framework}

A qualitative descriptive approach was used as a methodological orientation for this study. ${ }^{17}$ This approach was selected since the researcher aimed to stay close to the data and describe the personal experiences of the participants directly. 17 This study was set in five district hospitals in a rural health district in the KZN province in SA. All the therapists $(N=20)$ who were employed at the district hospitals were invited to participate in the FGDs and 17 out of 20 attended. Three therapists could not participate in the study as they had to attend to rural clinics away from the hospital at the time of data collection. Ethical clearance (Registration nr. 11/4/3) was obtained from the University of the Western Cape's Ethics committee, and permission to collect data was obtained from the KZN provincial Department of Health $(\mathrm{DOH})$ as well as all the relevant facility managers and heads of therapy departments. No persons other than the researcher and participants were present during data collection. The demographic details of the participants cannot be disclosed as it might compromise the anonymity of participants due to the small group of therapists that work in rural areas in the KZN province. An FGD was utilised as the instrument of data collection at each of the district hospitals. An FGD was deemed appropriate as it allowed for interactive sharing of experiences between more and less experienced therapists. ${ }^{18}$ The research question that was used as an opening question for the FGDs was 'What are your experiences with providing prosthetic rehabilitation in this rural setting?' This question was chosen based on the guidelines provided in the literature on conducting FGDs. 18

Five FGDs were conducted initially to describe the experiences of the therapists working in this setting. The data obtained from the initial five discussions were then analysed and themes and categories were derived. In order to establish credibility of the analysis and to explore the themes and categories derived from the first five FGDs in more depth, an additional two FGDs were conducted at two of the hospitals. Data saturation was reached after a total of seven FGDs. All FGDs were audio recorded. Field notes of personal impressions during the FGDs were taken by the researcher. The duration of the FGDs varied between 40 and $75 \mathrm{~min}$. Transcripts were returned via e-mail to the participants at three district hospitals for member checking and comments. Participants agreed that the transcripts were a true reflection of their statements and experiences.

\section{Data analysis}

Transcripts were manually coded using open coding and analysed by the researcher. Similar words were highlighted in the transcripts and grouped together to form a coherent idea or category. Keywords and phrases such as 'lack of' and 'shortage' were initially coded descriptively. These codes were then theoretically coded into more meaningful codes and subcategories such as 'lack of equipment' and 'staff shortages'. A comparative analysis was then performed to identify connections between different subcategories. For example, the subcategory of 'transdisciplinarity' was then linked to 'staff shortage' since even though they are inherently different they are strongly influenced by one another. Relevant subcategories were then analysed for similarity and grouped into main categories or themes. The three main categories or themes were then explored in more depth in the second round of 
FGDs. After the data analysis process was completed, the process of coding and thematic analysis was checked and verified by another researcher to ensure confirmability of the findings. Where there was a discrepancy in opinion, differences were discussed until consensus could be reached. The subsequent FGDs unanimously confirmed the categories identified, and no new categories were identified, indicating data saturation and the validity of the analysis. A process of back coding was then followed to identify the relevance of the main categories to one another, and the main themes were identified. A category was only included if it arose without prompting from the FGDs of at least two of the five hospitals and agreed upon by the entire group. The categories and themes were then also confirmed through and additional two FGDs to ensure the credibility of the analysis.

\section{Results}

The three main themes (Table 1) that arose from the five FGDs with the therapists in this district were (1) lack of support from the government health system, (2) poor socioeconomic status of patients and (3) cultural challenges. The main impact of these themes on patient care was the difficulty that therapists experienced in following up patients for pre-prosthetic rehabilitation after discharge from hospital.

\section{Poor support from the government health system}

The first three categories in this theme were inherently different, but could all be attributed to poor support from the government health system, which was the first theme that arose from the data.

Lack of equipment. A lack of resources or equipment for performing rehabilitation was discussed at length at all five of the initial FGDs. Lack of basic equipment such as metal hooks or clips for residuum bandages as well as assistive devices for mobility were noted. The lack of appropriate four-wheel drive vehicles to accommodate difficult terrain and follow up patients at home was also highlighted. Therapists mentioned there was a waiting period of at least 3 years for patients to receive a prosthesis:

Once it (the residuum) has been healed, you have to do the bandaging so that you leave the stump in a good position for it ... for the patient to be able to qualify for a leg, or something. So and we found that we don't even have those (residuum) bandages. (T16) 
Table I. Main themes resulting from the qualitative analysis.

\begin{tabular}{|c|c|c|}
\hline Supporting quotes & Categories & Themes \\
\hline $\begin{array}{l}\text { Once it (the stump) has been healed, you have to do the bandaging so that } \\
\text { you leave the stump in a good position for it ... for the patient to be able to } \\
\text { qualify for a leg, or something. So and we found that we don't even have those } \\
\text { (stump) bandages. (TI6) }\end{array}$ & $\begin{array}{l}\text { Lack of } \\
\text { equipment }\end{array}$ & $\begin{array}{l}\text { I. Poor support from } \\
\text { the government } \\
\text { health system }\end{array}$ \\
\hline $\begin{array}{l}\text { There have been critical times ... in the last month ... sometimes individual } \\
\text { professionals; they've not been, hmm ... supportive at the top structure } \\
\text { for therapy. With HR (human resources) ... I think that because we have X } \\
\text { number of therapists that means our component is sorted, we don't need } \\
\text { more (therapists). (TI) }\end{array}$ & Staff shortages & \\
\hline $\begin{array}{l}\text { I found that you not only a therapist in what you trained to be a therapist like } \\
\text { you not just a OT, like just a Physio, not just a Speech (therapist). You end up } \\
\text { doing social work, you end up doing speech therapy, you end up doing a bit of } \\
\text { dietetics if you can. (T8) }\end{array}$ & Transdisciplinarity & \\
\hline $\begin{array}{l}\text { Also patients expect ... if a patient comes to you ... their aim is they want } \\
\text { a grant no matter how much work you do, they're not going to get better } \\
\text { because they don't want to get better, they want to qualify for a grant. That is } \\
\text { the frustrating thing, because than, than it feels ... I often feel as if I'm doing this } \\
\text { job because I want to make people better and want to make them independent } \\
\text { and all they want is they want me to say shame, let's make you so that you can } \\
\text { qualify for a grant (LOL). (T6) }\end{array}$ & $\begin{array}{l}\text { Challenges with } \\
\text { disability grants }\end{array}$ & $\begin{array}{l}\text { 2. Poor } \\
\text { socioeconomic } \\
\text { circumstances of } \\
\text { patients }\end{array}$ \\
\hline $\begin{array}{l}\text {... it's really difficult to make follow-up appointments with someone who is } \\
\text { unemployed who will tell you no, I'll only have money on the end of the month, } \\
\text { you know ... so sometimes it's difficult ... (TI5) }\end{array}$ & $\begin{array}{l}\text { Lack of referral } \\
\text { and follow-up }\end{array}$ & \\
\hline $\begin{array}{l}\text { You can't separate traditional and cultural factors from your treatment, you can } \\
\text { do as much as you can in a hospital and if the family decides to go and consult } \\
\text { with a traditional healer. Your ... you can only advocate this much as to what is } \\
\text { good and what is bad from a medical or from our point of view. (TII) }\end{array}$ & Health beliefs & 3. Cultural factors \\
\hline $\begin{array}{l}\text { I remember there was this one day ... and you were there, ne? There was } \\
\text { someone who came here for physiotherapy so I took her file and wanted to } \\
\text { write on it and she could even kill me, cause she said ... you don't write there, } \\
\text { that is for the doctor. And when I asked who is the doctor then? And she said, } \\
\text { woman, you know. So that is the kind of perception that they'll have that ... } \\
\text { hey, you don't write on my file if you are, you know, my own race! (TI7) }\end{array}$ & Racial prejudice & \\
\hline
\end{tabular}

Staff shortages. Therapists highlighted the lack of permanent posts for therapists at the rural hospitals despite the large number of community service therapists who indicated that they would like to stay in a permanent capacity after the completion of their community service year. Therapists felt that they were short staffed and that the various hospitals' management (at the time of data collection in 2014) did not create posts when there were obvious staff shortages:

There have been critical times ... in the last month ... sometimes individual professionals; they've not been, hmm ... supportive at the top structure for therapy. With HR (human resources) ... I think that because we have $\mathrm{X}$ number of therapists that means our component is sorted, we don't need more (therapists). (T1)

Transdisciplinarity. Another concern for the majority of the therapists was the transdisciplinarity that naturally resulted from the staff shortages. Physiotherapists and occupational therapists, and sometimes even speech therapists, were required to perform the same duties, leading to a sense of loss of professional identity:

But it can also have a negative effect ... whereby, you know, you start ... you all kinda seen as one person ... you come out here (to work in a rural area), you lose your identity as an occupational therapist for example and you start to be seen as just a therapist ... and then 
everybody just start seeing you as this one person, you know ... the rehab team when we actually are different disciplines ... (T3)

I found that you not only a therapist in what you trained to be a therapist like you not just a OT, like just a Physio, not just a Speech (therapist). You end up doing social work, you end up doing speech therapy, you end up doing a bit of dietetics if you can. (T8)

\section{Poor socioeconomic circumstances of patients}

Challenges with disability grants. Even though therapists recognised the value of disability grants (DGs), they reported that it sometimes became a barrier to rehabilitation because patients would rather receive a grant than improve their functionality:

Also patients expect ... if a patient comes to you ... their aim is they want a grant no matter how much work you do, they're not going to get better because they don't want to get better, they want to qualify for a grant. That is the frustrating thing, because then, then it feels ... I often feel as if I'm doing this job because I want to make people better and want to make them independent and all they want is they want me to say shame, let's make you so that you can qualify for a grant. (T6)

Lack of referral and follow-up. The lack of patient referral and follow-up, and challenges relating to DGs gave rise to the theory that the poor socioeconomic status of patients in this specific context provides a big challenge for therapists.

The success of any rehabilitation programme is largely dependent on the patient's adherence to the programme and follow-up by the therapist. In this setting, therapists reported major obstacles in following up patients for rehabilitation and treatment:

... it's really difficult to make follow-up appointments with someone who is unemployed who will tell you no, I'll only have money on the end of the month, you know ... so sometimes it's difficult ... (T15)

... here people don't have money to come back for, say for instance, for an acute injury. We would like to see that patient maybe three times a week or once a week, but for this setting it's not possible. $(\mathrm{T} 7)$

\section{Cultural factors}

Finally, cultural factors arose as the third category from the last two subcategories: health beliefs and racial prejudice.

Health beliefs. Cultural beliefs impacted greatly on the rehabilitation services in this specific area and were often cited by therapists as a barrier to rehabilitation. The most common cultural belief that affected rehabilitation services was patients' belief regarding the cause of their disability. Patients in the rural areas often considered any type of disability the result of bewitchment or a curse and sought help from traditional healers: 
You can't separate traditional and cultural factors from your treatment, you can do as much as you can in a hospital and if the family decides to go and consult with a traditional healer. Your ... you can only advocate this much as to what is good and what is bad from a medical or from our point of view. (T11)

To go now [and advise the patient] you need to go do these exercises after you [the patient] think you've got this pain because you had this dream or you've been bewitched or something is a very, very challenging thing. (T9)

Racial prejudice. Communities also had a belief that therapists from their own community were inferior to therapists from other race groups:

And the other thing ... because we are black, people they undermine that. If you from the local area ... they say, oh, you know me ... it happens ... (T16)

I remember there was this one day ... and you were there, ne? There was someone who came here for physiotherapy so I took her file and wanted to write on it and she could even kill me, cause she said ... you don't write there, that is for the doctor. And when I asked who is the doctor then? And she said, woman, you know. So that is the kind of perception that they'll have that ... hey, you don't write on my file if you are, you know, my own race! (T17)

\section{Discussion}

\section{Lack of support from the government health system}

The lack of resources in the public health system in South Africa is not a new finding, but the extent of the deprivation of assistive devices and services in these hospitals are negatively affecting the patient outcomes. In this study, many patients were not rehabilitated for prosthetic fitting due to a lack of resources such as residuum bandaging or the binding clips that keep the bandage in place. The lack of a binding clip that costs as little as US\$0.25 prevented persons with a LLA the possible opportunity to receive a prosthetic limb. Therapists found this very frustrating. Another resource that negatively impacted on therapists' experience with providing prosthetic rehabilitation was the lack of four wheel drive vehicles in order to follow up patients at home after discharge. Therapists felt discouraged and overwhelmed by the lack of resources to perform adequate prosthetic rehabilitation.

The lack of qualified therapists and prosthetists to service the rural areas in South Africa is well documented.4,12,13,19 The contradiction in this setting was that posts were not being provided by the national $\mathrm{DOH}$ for people who want to work in this setting. The national DOH has implemented the compulsory community service programme in order to increase the number of therapists working in rural settings.7 According to the community service therapists who participated in the FGDs, they wanted to stay in a rural setting, but the necessary posts were not provided by the $\mathrm{DOH}$. The more experienced permanent therapists also highlighted that the resulting high turnover of new community service therapists each year negatively affected the continuity of rehabilitation services. 
The lack of permanent positions for therapy staff also resulted in a form of transdisciplinarity between Physiotherapists, Occupational therapists and sometimes even Speech therapists. This was a big concern for younger community service therapists. Transdisciplinary rehabilitation is a recognised approach to rehabilitation, specifically for children with disabilities. 20 Normally, two therapists from different professions would agree to work towards the same rehabilitation goal for a child. They would then teach each other selected discipline-specific skills that are necessary to achieve the specific goal. 20 In this study, the lack of human resources forced therapists to perform discipline-specific skills that were outside their scope of practice. Crossing the boundaries of the discipline-specific scopes of practice could also have negative legal implications if therapists are practising outside of their scope of practice and an injury to the patient occurs. Therapists were not working together on one patient; they were just performing the work of two different types of therapists. This is very challenging for newly qualified therapists, who are often not yet very confident in their own profession's skills. A negative community service experience also discourages medical professionals from remaining in the public health system. 6

The overriding theme of lack of support from the DOH seemed to keep reoccurring in relation to frustrations around patient follow-up after amputation surgery.

\section{Poor socioeconomic circumstances of patients}

Therapists reported that the DG was a challenge to pre-prosthetic rehabilitation in this specific setting. The South African Social Security Agency (SASSA) support persons with disabilities financially with an amount of US\$94 (R1500) on a monthly basis. ${ }^{21}$ Persons who might suffer a loss of income of at least 6 months due to a disability also qualify for a temporary DG. Persons who suffered the loss of a limb will immediately qualify for a DG due to the long waiting periods for a prosthesis. If they receive a prosthesis which would enable them to seek employment again, they might lose their DG. In this community, approximately $70 \%$ of the population survive on less than US\$50 (R800) per month. ${ }^{22}$ Only $12.7 \%$ of the population are employed and $65.8 \%$ are not economically active at all. 23 Understandably seeking rehabilitation might improve the patient's functional status, but result in a loss of income. Therapists were very frustrated as this impacted negatively on rehabilitation of persons with an LLA, but also highlighted the severity of the deprivation in this setting.

Poverty reinforces disability. 24 In this rural setting, a lack of finances was cited by the therapists as the main reason for lack of patient follow-up. Patients could not afford to access public transport in order to return for therapy. The lack of access to rehabilitation and health services calls for a decentralisation of health services in rural communities. Community-based care givers and rehabilitation care workers are being trained and employed in some provinces, but were not operational in this province at the time of data collection. At one hospital in this study, only one blind community care worker was employed to service a community of approximately 100,000 people. Again, therapists were 
highlighting these concerns specifically in relation to difficulty with post-operative rehabilitation and preparation for prosthetic fitting.

Investigating the feasibility of alternative approaches such as training more communitybased rehabilitation workers and tele-rehabilitation could potentially address some of these challenges.

\section{Cultural factors affecting rehabilitation services}

A finding that is potentially unique to this setting was the influence of cultural beliefs regarding the cause of disability on rehabilitation. According to the therapists, the belief that they were 'bewitched' affected patients' buy-in to Western therapy services. Patients believed that only a traditional healer or 'Sangoma' could reverse their disability. This finding is supported and can to some extent be explained by the Health Belief Model (HBM). 25 The HBM aims to explain why people do not engage in practices that could enhance their health. The four major constructs of perception in the HBM include perceived seriousness, perceived susceptibility, perceived benefit and perceived barriers. In this specific study sample, the perceived benefits of therapy were low due to cultural beliefs and the perceived barriers (cost and accessibility) too high. This negatively affected participation in preprosthetic rehabilitation where it was available and was considered a barrier to prosthetic fitting. Therapists who work in a rural setting tried to raise awareness of the causes of disability by doing health promotion in the community, but with little perceived success thus far. More emphasis should be placed on raising awareness of disability issues and the destigmatisation of disabilities in order to change people's health beliefs over time. Working together with and training traditional healers on when to refer patients to hospital could also assist to improve patients' trust in Western medicine.

Racial prejudice against medical professionals from their own community could also be explained by the HBM.25 People might believe that they will benefit more from the services of people who are not from their own community. This might only be an incidental finding, as this was not reported by all therapists, but only by some who worked in the more isolated and deep rural areas. The value of this finding could be to keep recruiting and training therapist from the rural areas, in order to change this misconception over time.

In this instance, cultural beliefs regarding the cause of disability and racial prejudices were identified by therapists as a barrier to providing prosthetic rehabilitation.

\section{Study limitations}

The findings of this study are generalisable to other rural districts in the same province, but not to other provinces in South Africa, as some of the challenges experienced by therapist were unique within their specific context. Due to the researcher's prior experience of working in this setting, the study findings might be biased even though steps were taken to ensure the credibility, transferability and confirmability of the findings. 


\section{Conclusion}

Pre-prosthetic rehabilitation is essential for successful fitting of a prosthesis. If a person with an LLA does not receive adequate rehabilitation after the loss of their limb, they are likely to develop complications of immobility such as joint contractures, a bulbous residuum and muscle weakness. Considering the long waiting periods and limited resources for prostheses in the rural areas of South Africa, these complications will further limit access to a prosthesis. There is an increasing demand for pre-prosthetic rehabilitation due to the increasing incidence of diabetes mellitus and its complications. In this rural context, there are many challenges to providing adequate rehabilitation for prosthetic fitting. The three main themes derived from the therapists' experiences in this rural context were poor support from the government health system, poor socioeconomic circumstances and cultural factors. Alternative strategies such as the implementation of a community-based rehabilitation approach to pre-prosthetic rehabilitation, training of traditional healers and exploring telerehabilitation options could address some of these barriers.

\section{Author contribution}

All authors contributed equally in the preparation of this manuscript.

\section{Declaration of conflicting interests}

The author(s) declared no potential conflicts of interest with respect to the research, authorship and/or publication of this article.

\section{Funding}

The author(s) disclosed receipt of the following financial support for the research, authorship, and/or publication of this article: This work was supported by National Research Foundation of South Africa (Grant/Award Number: 84336). 


\section{References}

1. Fredericks JP and Visagie S. The rehabilitation programme and functional outcomes of persons with lower limb amputations at a primary level rehabilitation centre. $S$ Afr J Occup Ther 2013; 43(3): 18-28.

2. Godlwana L. The impact of lower limb amputation on quality of life: a study done in the Johannesburg metropolitan area, South Africa. Unpublished Master's Thesis, University of the Witwatersrand, Johannesburg, South Africa, 2009.

3. World Health Organization (WHO). International classification of function, disability and health (ICF). Geneva: World Health Organization, 2001.

4. World Health Organization (WHO). World report on disability. Geneva: World Health Organization, 2011.

5. Kaplan SL. Outcome measurement and management: first steps for the practicing clinician. Philadelphia, PA: F.A. Davis Company, 2007

6. Ennion L, Johannesson A and Rhoda A. The use of a direct manufacturing prosthetic socket system in a rural community in South Africa: a pilot study and lessons for future research. Prosthet Orthot Int. Epub ahead of print 20 January 2017. DOI: 10.1177/0309364616683982.

7. Cooke R, Couper I and Versteeg M. Human resources for rural health. In: Gray A, Vawda Y and Jack C (eds) South African health review 2011. Durban, South Africa: Health Systems Trust, 2011, pp. 107-117.

8. Motala AA, Esterhuizen T, Gouws E, et al. Diabetes and other disorders of glycemia in a rural South African community: prevalence and associated risk factors. Diabetes Care 2008; 31: 1783-1788.

9. Eklund A. Prosthetics and orthotics programme guide: implementing $\mathrm{P} \& \mathrm{O}$ services in low-income settings, http:// www.ispoint.org/sites/default/files/img/programme_guide_ final_version.pdf (2006, accessed 29 June 2015).

10. Strait E. Prosthetics in developing countries, http://www. oandp.org/publications/resident/pdf/DevelopingCountries. pdf (2006, accessed 15 September 2015).

11. World Health Organization (WHO). Guidelines for training personnel in developing countries for prosthetic and orthotic services. Geneva: World Health Organization, 2005.

12. World Health Organization (WHO). Joint position paper on the provision of mobility devices in less-resourced settings: a step towards implementation of the Convention on the Rights of Persons with Disabilities related to personal mobility. Geneva: World Health Organization, 2011.

13. Gaede B and Versteeg M. The state of the right to health in rural South Africa (Chapter 9). In: Padarath A and English R (eds) South African health review, 2016, http://www. hst.org.za/publications/south-african-health-review-2011 (2011, accessed 22 March 2016). 14. Magnusson L. Prosthetic and orthotic services in developing countries. Dissertation, Jönköping, Jönköping University, 2014, https://www.divaportal.org/smash/get/diva2:754830/FULLTEXTo1.pdf

15. Stanmore E and Waterman H. Crossing professional and organizational boundaries: the implementation of generic rehabilitation assistants within three organizations in the northwest of England. Disabil Rehabil 2007; 29: 751-759. 
16. Pearlman J, Cooper RA, Krizack M, et al. Lower-limb prosthesis and wheelchairs in low income countries: an overview. IEEE Eng Med Biol 2008; 27(2): 12-22.

17. Sandelowski M. Whatever happened to qualitative description? Res Nurs Health 2000; 23(4): 334-340.

18. Morgan DL. Focus groups as qualitative research. 2nd ed. Thousand Oaks, CA: SAGE Publications, 2013.

19. Department of Health. HRH strategy for the health sector: 2012/13-2016/17, http://www.gov.za/sites/www.gov.za/ files/hrh_strategy_o.pdf (2011, accessed 3 May 2016).

20.York J, Rainforth B and Giangreco MF. Transdisciplinary teamwork and integrated therapy: clarifying the misconceptions. Pediatr Phys Ther 1990; 2(2): 73-79.

21. Government of South Africa. Disability grant, http://www.gov.za/services/socialbenefits/disability-grant (2016, accessed 15 August 2016).

22. South African Government. Department of cooperative governance and traditional affairs: Umkhanyakude district municipality profile, http://www.ukdm.gov.za/index.php/en/ investors/investor-profile (2011, accessed 11 June 2016).

23. Gwacela PS and Vilane SJ. Local government report: Umkhanyakude district municipality annual report 2010/2011, http://www.ukdm.gov.za/jdownloads/Annual\%20Reports/ annual_report_ukdm_-_2013_2014_fy_final_draft.pdf (2010/2011, accessed 6 May 2015). 24. Sala-i-Martin X. On the health-poverty trap. In: Lopez-Casasnovas G, Rivera B and Currais L (eds) Health and economic growth: findings and policy implications. Cambridge, MA: MIT Press, 2005.

25. Hochbaum GM. Health belief model, http://www.med. uottawa.ca/courses/epi6181/images/Health_Belief_Model_review.pdf (1958, accessed 13 August 2016). 